\title{
A FINITE TIME RESULT FOR VANISHING VISCOSITY IN THE PLANE WITH NONDECAYING VORTICITY*
}

\author{
ELAINE COZZI ${ }^{\dagger}$
}

\begin{abstract}
Assuming that initial velocity has finite energy and initial vorticity is bounded in the plane, we show that the unique solutions of the Navier-Stokes equations converge to the unique solution of the Euler equations in the $L^{\infty}$-norm uniformly over finite time as viscosity approaches zero. We also establish a rate of convergence.
\end{abstract}

Key words. Fluid mechanics, inviscid limit.

AMS subject classifications. 76D05, 76C99.

\section{Introduction}

We consider the Navier-Stokes equations modeling incompressible viscous fluid flow, given by

$$
(N S)\left\{\begin{array}{c}
\partial_{t} v_{\nu}+v_{\nu} \cdot \nabla v_{\nu}-\nu \Delta v_{\nu}=-\nabla p_{\nu} \\
\operatorname{div} v_{\nu}=0 \\
\left.v_{\nu}\right|_{t=0}=v_{\nu}^{0}
\end{array}\right.
$$

and the Euler equations modeling incompressible non-viscous fluid flow, given by

$$
(E)\left\{\begin{array}{c}
\partial_{t} v+v \cdot \nabla v=-\nabla p \\
\operatorname{div} v=0 \\
\left.v\right|_{t=0}=v^{0}
\end{array}\right.
$$

In this paper, we study the vanishing viscosity limit. The question of vanishing viscosity addresses whether or not a solution $v_{\nu}$ of $(N S)$ converges in some norm to a solution $v$ of $(E)$ with the same initial data as viscosity tends to 0 .

While there exist many vanishing viscosity results in the plane for weak solutions to the fluid equations, the most relevant result for our purposes is that of Chemin in [1] concerning Yudovich solutions to $(E)$. Specifically, in [8], Yudovich establishes the uniqueness of a solution $(v, p)$ to $(E)$ in the space $C\left(\mathbb{R}^{+} ; L^{2}\left(\mathbb{R}^{2}\right)\right) \times L_{l o c}^{\infty}\left(\mathbb{R}^{+} ; L^{2}\left(\mathbb{R}^{2}\right)\right)$ when $v^{0}$ belongs to $L^{2}\left(\mathbb{R}^{2}\right)$ and $\omega^{0}$ belongs to $L^{p}\left(\mathbb{R}^{2}\right) \cap L^{\infty}\left(\mathbb{R}^{2}\right)$ for some $p<\infty$. For this uniqueness class, Chemin proves that the vanishing viscosity limit holds in the $L^{p}$-norm uniformly over finite time, and he establishes a rate of convergence.

In this paper, we consider the case where initial velocity belongs to $L^{2}\left(\mathbb{R}^{2}\right)$, while initial vorticity is bounded and does not necessarily belong to $L^{p}\left(\mathbb{R}^{2}\right)$ for any $p<\infty$. The existence and uniqueness of solutions to $(E)$ with nondecaying vorticity and nondecaying velocity was proved by Serfati in [7]. Specifically, the author proves the following theorem.

THEOREM 1.1. Let $v^{0}$ and $\omega^{0}$ belong to $L^{\infty}\left(\mathbb{R}^{2}\right)$, and let $c \in \mathbb{R}$. For every $T>0$ there exists a unique solution $(v, p)$ to $(E)$ in the space $L^{\infty}\left([0, T] ; L^{\infty}\left(\mathbb{R}^{2}\right)\right) \times$

\footnotetext{
${ }^{*}$ Received: April 8, 2009; accepted (in revised version); November 4, 2009. Communicated by Francois Golse.

This material is based upon work supported by the Center for Nonlinear Analysis (CNA) under the National Science Foundation Grant No. DMS 0635983.

${ }^{\dagger}$ Department of Mathematical Sciences, Carnegie Mellon University, USA (ecozzi@andrew.cmu. edu).
} 
$L^{\infty}\left([0, T] ; C\left(\mathbb{R}^{2}\right)\right)$ with $\omega \in L^{\infty}\left([0, T] ; L^{\infty}\left(\mathbb{R}^{2}\right)\right), p(0)=c$, and with $\frac{p(t, x)}{|x|} \rightarrow 0$ as $|x| \rightarrow$ $\infty$.

The author also shows that the velocity satisfies the estimate

$$
\|v(t)\|_{L^{\infty}} \leq C|| v^{0} \|_{L^{\infty}} e^{C_{1}\left\|\omega^{0}\right\|_{L^{\infty} t}} .
$$

In [3], we show that for some $T_{0}>0$ the solutions to $(N S)$ with initial velocity and initial vorticity in $L^{\infty}\left(\mathbb{R}^{2}\right)$ converge in $L^{\infty}\left(\left[0, T_{0}\right] ; L^{\infty}\left(\mathbb{R}^{2}\right)\right)$ to the solutions of $(E)$ given by Theorem 1.1. This paper attempts to improve on the result of [3]. Specifically, in this paper we prove that if $v^{0}$ belongs to $L^{2}\left(\mathbb{R}^{2}\right)$ and $\omega^{0}$ belongs to $L^{\infty}\left(\mathbb{R}^{2}\right)$, then the unique solutions of the Navier-Stokes equations converge to the unique solution of the Euler equations in the $L^{\infty}$-norm uniformly over any finite time interval as viscosity approaches zero (see Theorem 3.1). Despite the different assumptions placed on initial velocity, the strategy for proving this result will follow the strategy of $[3]$.

We remark here that even though we assume that initial velocity belongs to $L^{2}\left(\mathbb{R}^{2}\right)$ rather than $L^{\infty}\left(\mathbb{R}^{2}\right)$, the boundedness of the initial vorticity combined with finite energy of the initial velocity imply that the initial velocity is bounded (see Lemma 2.1 and Remark 2.1). Therefore our solutions to $(E)$ satisfy the initial conditions given in Theorem 1.1. We can conclude from Theorem 1.1 that the velocity remains bounded as time evolves. Moreover, in section 2 we show that energy is conserved when $v^{0}$ is in $L^{2}\left(\mathbb{R}^{2}\right)$ and $\omega^{0}$ is in $L^{\infty}\left(\mathbb{R}^{2}\right)$, so that the solution $v$ to $(E)$ belongs to $L_{\text {loc }}^{\infty}\left(\mathbb{R}^{+} ; L^{2}\left(\mathbb{R}^{2}\right) \cap L^{\infty}\left(\mathbb{R}^{2}\right)\right)$.

In [3] we consider low, middle, and high frequencies of the difference between the solutions to $(N S)$ and $(E)$ separately. For both low and high frequencies we are able to show convergence in the $L^{\infty}$-norm on any finite time interval. For the middle frequencies, we are only able to show that convergence holds for short time. Our strategy for estimating the middle frequencies in [3] is to localize the frequencies of the vorticity formulations of $(N S)$ and $(E)$ and to consider the difference of the two resulting equations. We are then able to reduce the problem to showing that there exists $T_{0}>0$ such that the vanishing viscosity limit holds in the Besov space $\dot{B}_{\infty, \infty}^{0}\left(\mathbb{R}^{2}\right)$ uniformly on $\left[0, T_{0}\right]$.

We apply a similar approach here; however, in this case we use different methods along with the additional assumption that $v^{0} \in L^{2}\left(\mathbb{R}^{2}\right)$ to show that the vanishing viscosity limit holds in the $\dot{B}_{\infty, \infty}^{0}$-norm uniformly over any finite time interval. Combining this new estimate with the estimates from [3] for the low and high frequencies, we are able to extend our previous short time result to a finite time result. (In fact, the low frequency estimate with the additional assumption that the velocity has finite energy is considerably more straightforward than that in [3] and relies only on an application of Bernstein's Lemma and the membership of $v_{\nu}$ and $v$ to $L^{\infty}\left([0, T] ; L^{2}\left(\mathbb{R}^{2}\right)\right)$.)

In this paper, to estimate the $L^{\infty}$-norm of the middle frequency term $\left(S_{n}-\right.$ $\left.S_{-n}\right)\left(v_{\nu}-v\right)$, we initially follow the approach given in [3]. Specifically, we use the following estimate from [3]:

$$
\left\|\left(S_{n}-S_{-n}\right)\left(v_{\nu}-v\right)(t)\right\|_{L^{\infty}} \leq C n\left\|\left(v_{\nu}-v\right)(t)\right\|_{\dot{B}_{\infty, \infty}^{0}}
$$

for each $t>0$. We proceed to show that $v_{\nu}$ converges to $v$ in the $\dot{B}_{\infty, \infty}^{0}$-norm on any finite time interval as viscosity approaches 0 (see Lemma 3.3). It is in this proof that we deviate from [3] by replacing an argument using Gronwall's Lemma with an argument relying on Osgood's Lemma. We closely follow the proof in [1] which 
demonstrates that the vanishing viscosity limit holds in the energy norm uniformly over finite time when initial velocity belongs to $L^{2}\left(\mathbb{R}^{2}\right)$ and initial vorticity belongs to $L^{2}\left(\mathbb{R}^{2}\right) \cap L^{\infty}\left(\mathbb{R}^{2}\right)$.

The paper is organized as follows. In section 2, we discuss properties of solutions to $(E)$ with nondecaying vorticity. In section 3 and section 4 , we state and prove the main result; we devote section 4 entirely to showing that the vanishing viscosity limit holds in the $\dot{B}_{\infty, \infty}^{0}$-norm.

For background information on Littlewood-Paley theory, Bony's paraproduct decomposition, Besov spaces, and technical lemmas used throughout the paper, we refer the reader to section 2 of [3].

\section{Properties of solutions to the Euler equations with nondecaying vor- ticity}

In this section, we discuss properties of solutions to $(E)$ with nondecaying vorticity. We begin by stating a result giving Holder regularity of solutions to $(E)$ with initial velocity and initial vorticity in $L^{\infty}\left(\mathbb{R}^{2}\right)$. We refer the reader to section 3 of [3] for a proof of the Lemma.

Lemma 2.1. Let $v$ be the unique solution to (E) given by Theorem 1.1 with bounded initial vorticity and bounded initial velocity. Then the following estimate holds:

$$
\|v(t)\|_{C_{*}^{1}} \leq C\left\|v^{0}\right\|_{L^{\infty}} e^{C_{1}\left\|\omega^{0}\right\|_{L} \infty t}+C\left\|\omega^{0}\right\|_{L^{\infty}} .
$$

We now address the question of energy conservation when $\omega^{0}$ belongs to $L^{\infty}\left(\mathbb{R}^{2}\right)$ and $v^{0}$ belongs to $L^{2}\left(\mathbb{R}^{2}\right)$. As mentioned in section 1 , with these assumptions on the initial data Theorem 1.1 guarantees that the solution $v$ to $(E)$ belongs to $L_{\text {loc }}^{\infty}\left(\mathbb{R}^{+} ; L^{\infty}\left(\mathbb{R}^{2}\right)\right)$. However, in [7] the author does not assume $v^{0} \in L^{2}\left(\mathbb{R}^{2}\right)$ and therefore does not address the issue of energy conservation when vorticity is nondecaying. For this we prove the following lemma.

Lemma 2.2. Assume $v^{0}$ belongs to $L^{2}\left(\mathbb{R}^{2}\right)$ and $\omega^{0}$ belongs to $L^{\infty}\left(\mathbb{R}^{2}\right)$. Then the solution to $(E)$ given by Theorem 1.1 satisfies $\|v(t)\|_{L^{2}}=\left\|v^{0}\right\|_{L^{2}}$ for all $t>0$.

Proof. In [7], when the author proves existence of a solution $(v, p)$ to $(E)$ satisfying the assumptions of Theorem 1.1, he assumes $v^{0}$ and $\omega^{0}$ belong to $L^{\infty}\left(\mathbb{R}^{2}\right)$, and he uses the sequence $\left\{S_{n} v^{0}\right\}$ to generate a sequence of smooth solutions $\left\{\left(v_{n}, p_{n}\right)\right\}$ to $(E)$ with initial velocity $S_{n} v^{0}$. The author then proceeds to prove that the sequence $\left\{v_{n}\right\}$ is uniformly bounded in $W_{l o c}^{1, p}\left([0, T] \times \mathbb{R}^{2}\right)$ for $p \in(2+\epsilon, \infty), \epsilon>0$. Using a standard diagonalization argument, one can construct a subsequence, which we henceforth denote as $\left\{v_{n}\right\}$, converging to $v$ in $W_{l o c}^{1, q}\left([0, T] \times \mathbb{R}^{2}\right)$ for $q<p$. To complete the proof, the author passes to the limit in $(E)$ written in weak form to show that $(v, p)$ solves $(E)$. (We refer the reader to [7] for the details of this argument.)

To prove Lemma 2.2, we use the same sequence $\left\{v_{n}\right\}$ of smooth solutions converging in $W_{l o c}^{1, q}\left([0, T] \times \mathbb{R}^{2}\right)$ as that utilized by Serfati, but we now also assume that $v^{0}$ belongs to $L^{2}\left(\mathbb{R}^{2}\right)$. In this case we have, for each $t>0$ and for each $n$,

$$
\left\|v_{n}(t)\right\|_{L^{2}}=|| S_{n} v^{0}\left\|_{L^{2}} \leq C\right\| v^{0} \|_{L^{2}},
$$

where $C$ is an absolute constant. Therefore, for each fixed $t$, there exists a subsequence $\left\{v_{n_{k}}(t)\right\}$ converging weakly in $L^{2}\left(\mathbb{R}^{2}\right)$ to $\bar{v}(t)$ with $\|\bar{v}(t)\|_{L^{2}} \leq C\left\|v^{0}\right\|_{L^{2}}$. Moreover, by the preceding paragraph, $\left\{v_{n}\right\}$ converges to the solution $v$ of $(E)$ in $L_{l o c}^{2}\left([0, T] \times \mathbb{R}^{2}\right)$, implying that $\left\{v_{n_{k}}(t)\right\}$ converges to $v(t)$ weakly in $L^{2}(\Omega)$ for each bounded set $\Omega \subset \mathbb{R}^{2}$ 
and for almost every $t \in[0, T]$. Using uniqueness of weak limits we can conclude that $\bar{v}(t)=v(t)$ for almost every $t$ in $[0, T]$; thus the weak solution $v$ generated from initial data $v^{0}$ belongs to $L_{l o c}^{\infty}\left(\mathbb{R}^{+} ; L^{2}\left(\mathbb{R}^{2}\right) \cap L^{\infty}\left(\mathbb{R}^{2}\right)\right)$.

To complete the proof of Lemma 2.2, we use a result proved by Duchon and Robert in [4]. In [4], the authors show that weak solutions $v$ to $(E)$ in $L_{l o c}^{3}\left(\mathbb{R}^{+} ; L^{3}\left(\mathbb{R}^{2}\right)\right)$ conserve energy if

$$
D_{\epsilon}(v)(t, x)=\frac{1}{4} \int_{\mathbb{R}^{2}} \nabla \phi^{\epsilon}(y) \cdot \delta v(t)(\delta v(t))^{2} d y
$$

approaches 0 in $\mathcal{D}^{\prime}\left((0, T) \times \mathbb{R}^{2}\right)$ as $\epsilon$ approaches 0 , where $\phi$ is a standard mollifier on $\mathbb{R}^{2}, \phi^{\epsilon}(y)=\frac{1}{\epsilon^{2}} \phi\left(\frac{y}{\epsilon}\right)$, and $\delta v(t)=v(t, x+y)-v(t, x)$.

To see that $\frac{1}{4} \int_{\mathbb{R}^{2}} \nabla \phi^{\epsilon}(y) \cdot \delta v(t)(\delta v(t))^{2} d y$ approaches 0 for the solutions in Lemma 2.2 , we utilize the membership of $v$ to $L_{l o c}^{\infty}\left(\mathbb{R}^{+} ; C_{*}^{1}\left(\mathbb{R}^{2}\right)\right)$. Taking into account the support of $\phi$, we can write

$$
\begin{aligned}
\left|\int_{\mathbb{R}^{2}} \nabla \phi^{\epsilon}(y) \cdot \delta v(t)(\delta v(t))^{2} d y\right| & \leq C \int_{\mathbb{R}^{2}} \frac{\epsilon}{|y|^{2}}\left|\frac{1}{\epsilon^{2}}(\nabla \phi)\left(\frac{y}{\epsilon}\right) \cdot \delta v(t)(\delta v(t))^{2}\right| d y \\
& \leq C \epsilon \|\left. v(t)\right|_{C^{\frac{2}{3}}} ^{3} \leq\left(C e^{C_{1} t}\right) \epsilon
\end{aligned}
$$

where we used Lemma 2.1 to get the last inequality, and $C$ and $C_{1}$ depend only on the initial data. This completes the proof of Lemma 2.2.

REMARK 2.1. We remark here that we can revise the proof of Lemma 2.1 from [3] to show that

$$
\|v(t)\|_{C_{*}^{1}} \leq C|| v^{0}\left\|_{L^{2}}+C\right\| \omega^{0} \|_{L^{\infty}}
$$

To do this we simply apply Gronwall's Lemma and Young's inequality to the low frequencies, and use energy conservation to get the series of inequalities $\left\|S_{0} v(t)\right\|_{L^{\infty}} \leq$ $C\|v(t)\|_{L^{2}}=C\left\|v^{0}\right\|_{L^{2}}$. We also point out that an estimate identical to (2.2) holds for $v_{\nu}$ by the argument given in the proof of Lemma 2.1 combined with the fact that the energy of Leray solutions to $(N S)$ is nonincreasing over time.

Finally, we will need a uniform bound in time on the $L^{\infty}$-norms of the vorticities corresponding to the solutions of $(N S)$ and $(E)$. For fixed $\nu \geq 0$, we have that

$$
\left\|\omega_{\nu}(t)\right\|_{L^{\infty}} \leq\left\|\omega_{\nu}^{0}\right\|_{L^{\infty}}
$$

for all $t \geq 0$. One can prove this bound by applying the maximum principle to the vorticity formulations of $(N S)$ and $(E)$. We refer the reader to Lemma 3.1 of [6] for a detailed proof.

\section{Statement and proof of the main result}

We are now prepared to state the main theorem.

Theorem 3.1. Let $v_{\nu}$ be the unique Leray solution to (NS) and let $v$ be the unique solution to (E), both with initial data $v^{0}$ in $L^{2}\left(\mathbb{R}^{2}\right)$ and $\omega^{0}$ in $L^{\infty}\left(\mathbb{R}^{2}\right)$. Then there exist constants $C$ and $C_{1}$, depending only on the initial data, such that the following estimate holds for any fixed $T>0$ and for any $\alpha \in(0,1)$ :

$$
\left\|v_{\nu}-v\right\|_{L^{\infty}\left([0, T] ; L^{\infty}\left(\mathbb{R}^{2}\right)\right)} \leq C(T+1)(\sqrt{\nu})^{\alpha e^{-C_{1} T}} .
$$


Proof. To establish the result, we apply a similar strategy to that used to prove Theorem 3 in [3]. We let $v_{\nu}$ and $v$ be the unique solutions to $(N S)$ and $(E)$, respectively, satisfying the assumptions of Theorem 3.1. In what follows, we let $v_{n}=$ $S_{n} v$ and $\omega_{n}=S_{n} \omega(v)$. We have the following inequality:

$$
\begin{aligned}
& \left\|v_{\nu}-v\right\|_{L^{\infty}\left([0, T] ; L^{\infty}\left(\mathbb{R}^{2}\right)\right)} \leq\left\|S_{-n}\left(v_{\nu}-v\right)\right\|_{L^{\infty}\left([0, T] ; L^{\infty}\left(\mathbb{R}^{2}\right)\right)} \\
& \quad+\left\|\left(I d-S_{-n}\right)\left(v_{\nu}-v_{n}\right)\right\|_{L^{\infty}\left([0, T] ; L^{\infty}\left(\mathbb{R}^{2}\right)\right)} \\
& \quad+\left\|\left(I d-S_{-n}\right)\left(v_{n}-v\right)\right\|_{L^{\infty}\left([0, T] ; L^{\infty}\left(\mathbb{R}^{2}\right)\right)} .
\end{aligned}
$$

We will estimate each of the three terms on the right hand side of the inequality in (3.2). We begin with the third term. We use the definition of $v_{n}$, Bernstein's Lemma, Lemma 3 of [3] (this Lemma gives the bound $\left\|\dot{\Delta}_{j} \nabla v\right\|_{L^{\infty}} \leq C\left\|\dot{\Delta}_{j} \omega\right\|_{L^{\infty}}$ for all $j \in \mathbb{Z}$ ), and (2.3) to obtain the inequality

$$
\left\|\left(I d-S_{-n}\right)\left(v_{n}-v\right)\right\|_{L^{\infty}\left([0, T] ; L^{\infty}\left(\mathbb{R}^{2}\right)\right)} \leq C 2^{-n}\left\|\omega^{0}\right\|_{L^{\infty}}
$$

for any $T>0$. To bound the first term on the right hand side of (3.2), we use the fact that the Fourier transform of $S_{-n}\left(v_{\nu}-v\right)$ is supported on a ball of radius $2^{-n}$, along with Lemma 2.2 and Bernstein's Lemma, to conclude that

$$
\left\|S_{-n}\left(v_{\nu}-v\right)(t)\right\|_{L^{\infty}} \leq C 2^{-n}\left\|S_{-n}\left(v_{\nu}-v\right)(t)\right\|_{L^{2}} \leq C 2^{-n}\left\|v^{0}\right\|_{L^{2}}
$$

for every $t>0$. It remains to bound the second term on the right hand side of (3.2), given by $\left\|\left(I d-S_{-n}\right)\left(v_{\nu}-v_{n}\right)\right\|_{L^{\infty}\left([0, T] ; L^{\infty}\left(\mathbb{R}^{2}\right)\right)}$. We prove the following proposition.

Proposition 3.1. Let $v_{\nu}$ and $v$ be solutions to $(N S)$ and $(E)$, respectively, satisfying the properties of Theorem 3.1. Then there exist constants $C$ and $C_{1}$, depending only on the initial data, such that the following estimate holds for any fixed $\alpha \in(0,1)$ and $T>0$, and for sufficiently large $n$ :

$$
\left\|\left(I d-S_{-n}\right)\left(v_{\nu}-v_{n}\right)\right\|_{L^{\infty}\left([0, T] ; L^{\infty}\left(\mathbb{R}^{2}\right)\right)} \leq C(T+1) 2^{-n \alpha e^{-C_{1} T}} .
$$

Remark 3.2. In the proof of Proposition 3.1, we let $\nu=2^{-2 n}$. Therefore, the dependence of the right hand side of (3.5) on $\nu$ is hidden in its dependence on $n$.

Proof. As in the proof of Proposition 7 in [3], we have that

$$
\left\|\left(I d-S_{-n}\right)\left(v_{\nu}-v_{n}\right)(t)\right\|_{L^{\infty}} \leq C 2^{-n}\left\|\omega^{0}\right\|_{L^{\infty}}+C n\left\|\left(v_{\nu}-v_{n}\right)(t)\right\|_{\dot{B}_{\infty, \infty}^{0}} .
$$

To bound $\|\left.\left(v_{\nu}-v_{n}\right)(t)\right|_{\dot{B}_{\infty, \infty}^{0}}$, we need the following lemma, whose proof we postpone until the next section.

Lemma 3.3. Let $v_{\nu}$ and $v$ be solutions to $(N S)$ and $(E)$, respectively, satisfying the properties of Theorem 3.1. Then there exist constants $C$ and $C_{1}$, depending only on the initial data, such that the following estimate holds for any fixed $\alpha \in(0,1)$ and $T>0$ :

$$
\left\|v_{\nu}-v_{n}\right\|_{L^{\infty}\left([0, T] ; \dot{B}_{\infty, \infty}^{0}\left(\mathbb{R}^{2}\right)\right)} \leq C(T+1) 2^{-n \alpha e^{-C_{1} T}} .
$$

Since $\alpha \in(0,1)$ is arbitrary, we can write

$$
n \| v_{\nu}-\left.v_{n}\right|_{L^{\infty}\left([0, T] ; \dot{B}_{\infty, \infty}^{0}\left(\mathbb{R}^{2}\right)\right)} \leq C(T+1) 2^{-n \alpha e^{-C_{1} T}}
$$


for sufficiently large $n$. Combining this estimate with the estimate given in (3.6) yields Proposition 3.1.

To complete the proof of Theorem 3.1, we combine (3.2), (3.4), Proposition 3.1, and (3.3) to get the following estimate for fixed $T>0$ and for sufficiently large $n$ :

$$
\left\|\left(v_{\nu}-v\right)\right\|_{L^{\infty}\left([0, T] ; L^{\infty}\left(\mathbb{R}^{2}\right)\right)} \leq C(T+1) 2^{-n \alpha e^{-C_{1} T}} .
$$

Using the equality $n=-\frac{1}{2} \log _{2} \nu$, we obtain (3.1). This completes the proof of Theorem 3.1. We devote the next section to the proof of Lemma 3.3.

\section{Proof of Lemma 3.3}

We begin with some notation. For the proof of Lemma 3.3, we let $\bar{\omega}_{n}=\omega_{\nu}-\omega_{n}$ and $\bar{v}_{n}=v_{\nu}-v_{n}$.

We recall the following estimate, which is proved in section 5 of [3]:

$$
\begin{aligned}
\partial_{t}\left\|\bar{v}_{n}(t)\right\|_{\dot{B}_{\infty, \infty}^{0} \leq} \leq & C\left(\left\|\bar{v}_{n} \omega_{\nu}(t)\right\|_{\dot{B}_{\infty, \infty}^{0}}+\left\|\nu \nabla \omega_{n}(t)\right\|_{\dot{B}_{\infty, \infty}^{0}}\right. \\
& \left.+\left\|\tau_{n}(v, \omega)(t)\right\|_{\dot{B}_{\infty, \infty}^{0}}+\sup _{j \in \mathbb{Z}} 2^{-j}\left\|\left[\dot{\Delta}_{j}, v_{n} \cdot \nabla\right] \bar{\omega}_{n}(t)\right\|_{L^{\infty}}\right),
\end{aligned}
$$

where

$$
\tau_{n}(v, \omega)=r_{n}(v, \omega)-\left(v-v_{n}\right)\left(\omega-\omega_{n}\right)
$$

and

$$
r_{n}(v, \omega)=\int \check{\psi}(y)\left(v\left(x-2^{-n} y\right)-v(x)\right)\left(\omega\left(x-2^{-n} y\right)-\omega(x)\right) d y .
$$

We observe that

$$
\left\|\bar{v}_{n}(t)\right\|_{\dot{B}_{\infty, \infty}^{0}} \leq C\left\|\bar{v}_{n}(t)\right\|_{L^{\infty}} \leq C\left(\left\|v^{0}\right\|_{L^{2}}+\left\|\omega^{0}\right\|_{L^{\infty}}\right)
$$

by Remark 2.1, and we define $A=1+C\left(\left\|v^{0}\right\|_{L^{2}}+\left\|\omega^{0}\right\|_{L^{\infty}}\right)$ with $C$ as in Remark 2.1. If we define $\delta_{n}(t)$ by

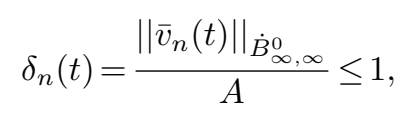

then, dividing both sides of (4.1) by $A$, we see that

$$
\begin{aligned}
\partial_{t} \delta_{n}(t) \leq & \frac{C}{A}\left(\left\|\bar{v}_{n} \omega_{\nu}(t)\right\|_{\dot{B}_{\infty, \infty}^{0}}+\left\|\nu \nabla \omega_{n}(t)\right\|_{\dot{B}_{\infty, \infty}^{0}}\right. \\
& \left.+\left\|\tau_{n}(v, \omega)(t)\right\|_{\dot{B}_{\infty, \infty}^{0}}+\sup _{j \in \mathbb{Z}} 2^{-j}\left\|\left[\dot{\Delta}_{j}, v_{n} \cdot \nabla\right] \bar{\omega}_{n}(t)\right\|_{L^{\infty}}\right) .
\end{aligned}
$$

To complete the proof of Lemma 3.3, we estimate each term on the right hand side of (4.2). The strategies for estimating the terms $\left\|\bar{v}_{n} \omega_{\nu}(t)\right\|_{\dot{B}_{\infty, \infty}^{0}}, \nu\left\|\nabla \omega_{n}(t)\right\|_{\dot{B}_{\infty, \infty}^{0}}$, and $\left\|\tau_{n}(v, \omega)(t)\right\|_{\dot{B}_{\infty, \infty}^{0}}$ are virtually identical to those in [3]; however, with the additional assumption that $v \in L^{\infty}\left([0, T] ; L^{2}\left(\mathbb{R}^{2}\right)\right)$, we are able to to use (2.2) in place of Lemma 1.1 when bounding $\|v(t)\|_{C^{\alpha}}$ for $\alpha<1$. This improvement applied to the proofs of the estimates in [3] for these terms yields the inequalities

$$
\left\|\bar{v}_{n}(t)\right\|_{L^{\infty}} \leq C 2^{-n}+C\left\|\left(S_{n}-S_{-n}\right)\left(v_{\nu}-v_{n}\right)(t)\right\|_{L^{\infty}},
$$


as well as

$$
\begin{aligned}
\left\|\bar{v}_{n} \omega_{\nu}(t)\right\|_{\dot{B}_{\infty, \infty}^{0}} & \leq C 2^{-n}+C\left\|\left(S_{n}-S_{-n}\right)\left(v_{\nu}-v_{n}\right)(t)\right\|_{L^{\infty}}, \\
\nu\left\|\nabla \omega_{n}(t)\right\|_{\dot{B}_{\infty, \infty}^{0}} & \leq C 2^{-n}\left\|\omega^{0}\right\|_{L^{\infty}}, \text { and } \\
\left\|\tau_{n}(v, \omega)(t)\right\|_{\dot{B}_{\infty, \infty}^{0}} & \leq C 2^{-n \alpha},
\end{aligned}
$$

where the first inequality of (4.4) follows from (4.3). We remark that we let $\nu=2^{-2 n}$ in the second inequality of (4.4).

Combining (4.2) with (4.4) gives

$$
\partial_{t} \delta_{n}(t) \leq \frac{C}{A}\left(2^{-n \alpha}+\left\|\left(S_{n}-S_{-n}\right)\left(v_{\nu}-v_{n}\right)(t)\right\|_{L^{\infty}}+\sup _{j \in \mathbb{Z}} 2^{-j}\left\|\left[\dot{\Delta}_{j}, v_{n} \cdot \nabla\right] \bar{\omega}_{n}(t)\right\|_{L^{\infty}}\right),
$$

where $C$ depends only on the initial data. To estimate $\left\|\left(S_{n}-S_{-n}\right)\left(v_{\nu}-v_{n}\right)(t)\right\|_{L^{\infty}}$, we let $p, q \in(1, \infty)$ satisfy $\frac{1}{p}+\frac{1}{q}=1$, and we observe that

$$
\begin{aligned}
& \left\|\left(S_{n}-S_{-n}\right)\left(v_{\nu}-v_{n}\right)(t)\right\|_{L^{\infty}} \\
\leq & \sum_{j=-n}^{0}\left\|\dot{\Delta}_{j}\left(\bar{v}_{n}\right)(t)\right\|_{L^{\infty}}^{\frac{1}{p}}\left\|\dot{\Delta}_{j}\left(\bar{v}_{n}\right)(t)\right\|_{L^{\infty}}^{\frac{1}{q}} \\
& +\sum_{j=1}^{n}\left\|\dot{\Delta}_{j}\left(\bar{v}_{n}\right)(t)\right\|_{L^{\infty}}^{\frac{1}{p}}\left\|\dot{\Delta}_{j}\left(\bar{v}_{n}\right)(t)\right\|_{L^{\infty}}^{\frac{1}{q}} \\
\leq & \left\|\bar{v}_{n}(t)\right\|_{\dot{B}_{\infty, \infty}^{0}}^{\frac{1}{q}}\left(\sum_{j=-n}^{0} 2^{\frac{j}{p}}\left\|\dot{\Delta}_{j}\left(\bar{v}_{n}\right)(t)\right\|_{L^{2}}^{\frac{1}{p}}+\sum_{j=1}^{n} 2^{\frac{-j}{p}}\left\|\bar{v}_{n}(t)\right\|_{\dot{B}_{\infty, \infty}^{1}}^{\frac{1}{p}}\right) \\
\leq & C\left\|\bar{v}_{n}(t)\right\|_{\dot{B}_{\infty, \infty}^{0}}^{\frac{1}{q}} \frac{1}{1-2^{-\frac{1}{p}}}\left(\left\|v^{0}\right\|_{L^{2}}^{\frac{1}{p}}+\left\|\bar{v}_{n}(t)\right\|_{\dot{B}_{\infty, \infty}^{1}}^{\frac{1}{p}}\right) \leq C p\left\|\bar{v}_{n}(t)\right\|_{\dot{B}_{\infty, \infty}^{0}}^{\frac{1}{q}} .
\end{aligned}
$$

Here we used Bernstein's Lemma to get the second inequality and Lemma 2.2 to get the third inequality. For the last inequality, we bounded $\left\|\bar{v}_{n}(t)\right\|_{\dot{B}_{\infty, \infty}^{1}}$ with $C|| \omega^{0} \|_{L^{\infty}}$ using properties of homogeneous Besov spaces (see [3]), Lemma 3 of [3], and (2.3), and we bounded $\frac{1}{1-2^{-\frac{1}{p}}}$ with $C p$, where $C$ is an absolute constant. Combining (4.6) and (4.5) gives

$$
\begin{aligned}
\partial_{t} \delta_{n}(t) & \leq \frac{C}{A}\left(2^{-n \alpha}+p\left\|\bar{v}_{n}(t)\right\|_{\dot{B}_{\infty, \infty}^{0}}^{\frac{1}{q}}+\sup _{j \in \mathbb{Z}} 2^{-j}\left\|\left[\dot{\Delta}_{j}, v_{n} \cdot \nabla\right] \bar{\omega}_{n}(t)\right\|_{L^{\infty}}\right) \\
& \leq \frac{C}{A} 2^{-n \alpha}+\frac{C p}{A^{\frac{1}{p}}} \delta_{n}(t)^{\frac{1}{q}}+\frac{C}{A} \sup _{j \in \mathbb{Z}} 2^{-j}\left\|\left[\dot{\Delta}_{j}, v_{n} \cdot \nabla\right] \bar{\omega}_{n}(t)\right\|_{L^{\infty}} .
\end{aligned}
$$

Since $A \geq 1$, we can write

$$
\partial_{t} \delta_{n}(t) \leq C 2^{-n \alpha}+C p \delta_{n}(t)^{\frac{1}{q}}+\frac{C}{A} \sup _{j \in \mathbb{Z}} 2^{-j}\left\|\left[\dot{\Delta}_{j}, v_{n} \cdot \nabla\right] \bar{\omega}_{n}(t)\right\|_{L^{\infty}}
$$

It remains to bound the commutator term on the right hand side of (4.7). We prove the following lemma.

LEMMA 4.1. Let $v$ be the unique solution to (E) satisfying the conditions of Theorem 3.1. Then the following estimate holds for any pair $(p, q) \in(1, \infty) \times(1, \infty)$ satisfying 
$\frac{1}{p}+\frac{1}{q}=1:$

$$
\sup _{j \in \mathbb{Z}} 2^{-j}\left\|\left[\dot{\Delta}_{j}, v_{n} \cdot \nabla\right] \bar{\omega}_{n}(t)\right\|_{L^{\infty}} \leq C\left(2^{-n}+p\left\|\bar{v}_{n}(t)\right\|_{\dot{B}_{\infty, \infty}^{0}}^{\frac{1}{q}}\right),
$$

where $C$ depends only on $\left\|v^{0}\right\|_{L^{\infty}}$ and $\left\|\omega^{0}\right\|_{L^{\infty}}$.

Proof. Throughout the proof, to simplify notation we will often omit the time variable $t$. When $t$ is omitted from a calculation, it is assumed that $t$ is fixed throughout that calculation.

The proof of Lemma 4.1 is similar to the proof of Lemma 10 in [3], the main difference being the bound on $\left\|\bar{v}_{n}(t)\right\|_{L^{\infty}}$ coming from (4.3) and (4.6). For this reason, we refer to [3] throughout the proof for various estimates. We first use Bony's paraproduct decomposition to write

$$
\left[\dot{\Delta}_{j}, v_{n} \cdot \nabla\right] \bar{\omega}_{n}=\sum_{m=1}^{2}\left[\dot{\Delta}_{j}, T_{v_{n}^{m}} \partial_{m}\right] \bar{\omega}_{n}+\left[\dot{\Delta}_{j}, T_{\partial_{m}} \cdot v_{n}^{m}\right] \bar{\omega}_{n}+\left[\dot{\Delta}_{j}, \partial_{m} R\left(v_{n}^{m}, \cdot\right)\right] \bar{\omega}_{n}
$$

To bound the $L^{\infty}$-norm of the first term on the right hand side, we consider the cases $j<0$ and $j \geq 0$ separately. For $j \geq 0$, we use the definition of the paraproduct and properties of the partition of unity to establish

$$
\left[\dot{\Delta}_{j}, T_{v_{n}} \partial_{m}\right]=\sum_{j^{\prime}=\max \{1, j-4\}}^{j+4}\left[\dot{\Delta}_{j}, S_{j^{\prime}-1}\left(v_{n}\right)\right] \Delta_{j^{\prime}} \partial_{m}
$$

We then express the operator $\dot{\Delta}_{j}$ as a convolution with $\check{\varphi}_{j}$, write out the commutator on the right hand side of (4.9), and change variables. This yields

$$
\begin{aligned}
& \left\|\left[\dot{\Delta}_{j}, T_{v_{n}} \partial_{m}\right] \bar{\omega}_{n}\right\|_{L^{\infty}} \\
\leq & \sum_{j^{\prime}=\max \{1, j-4\}}^{j+4} \| \int \check{\varphi}(y)\left(S_{j^{\prime}-1} v_{n}\left(x-2^{-j} y\right)\right. \\
& \left.-S_{j^{\prime}-1} v_{n}(x)\right) \Delta_{j^{\prime}} \partial_{m} \bar{\omega}_{n}\left(x-2^{-j} y\right) d y \|_{L^{\infty}} \\
\leq & \sum_{j^{\prime}=\max \{1, j-4\}}^{j+4} 2^{j^{\prime}-j}\left\|S_{j^{\prime}-1} \nabla v_{n}\right\|_{L^{\infty}}\left\|\Delta_{j^{\prime}} \bar{\omega}_{n}\right\|_{L^{\infty}} \int|\check{\varphi}(y) \| y| d y \\
\leq & C\left\|\bar{v}_{n}\right\|_{\dot{B}_{\infty, \infty}^{0}}^{\frac{1}{q}} \sum_{j^{\prime}=\max \{1, j-4\}}^{j+4}\left(2^{j^{\prime}-j} 2^{j^{\prime}}\left\|\Delta_{j^{\prime}} \bar{v}_{n}\right\|_{L^{\infty}}^{\frac{1}{p}} \sum_{l=-1}^{j^{\prime}-2}\left\|\Delta_{l} \nabla v_{n}\right\|_{L^{\infty}}\right) .
\end{aligned}
$$

We bound $\left\|\Delta_{l} \nabla v_{n}\right\|_{L^{\infty}}$ by $\|v\|_{L^{2}}+\|\omega\|_{L^{\infty}}$ for each $l$ using Remark 2.1 and we bound $\left\|\Delta_{j^{\prime}} \bar{v}_{n}\right\|_{L^{\infty}}^{\frac{1}{p}}$ by $2^{\frac{-j^{\prime}}{p}}\left\|\Delta_{j^{\prime}} \bar{\omega}_{n}\right\|_{L^{\infty}}^{\frac{1}{p}}$ using Bernstein's Lemma. We can then write

$$
\begin{aligned}
& \quad\left\|\left[\dot{\Delta}_{j}, T_{v_{n}} \partial_{m}\right] \bar{\omega}_{n}\right\|_{L^{\infty}} \\
& \leq\left\|\bar{v}_{n}\right\|_{\dot{B}_{\infty, \infty}^{0}}^{\frac{1}{q}}\left(\|v\|_{L^{2}}+\|\omega\|_{L^{\infty}}\right) \\
& \quad \times \sum_{j^{\prime}=\max \{1, j-4\}}^{j+4}\left(2^{j^{\prime}-j} 2^{j^{\prime}}\left\|\Delta_{j^{\prime}} \bar{\omega}_{n}\right\|_{L^{\infty}}^{\frac{1}{p}} \sum_{l \leq j^{\prime}} 2^{\frac{l-j^{\prime}}{p}} 2^{\frac{-l}{p}}\right) \\
& \leq C 2^{j}\left\|\bar{v}_{n}\right\|_{\dot{B}_{\infty, \infty}^{0}}^{\frac{1}{q}}\left(\|v\|_{L^{2}}+\|\omega\|_{L^{\infty}}\right)\left\|\bar{\omega}_{n}\right\|_{L^{\infty}}^{\frac{1}{p}} \frac{1}{1-2^{-\frac{1}{p}}} .
\end{aligned}
$$


We bound $\|v\|_{L^{2}},\|\omega\|_{L^{\infty}}$, and $\left\|\bar{\omega}_{n}\right\|_{L^{\infty}}^{\frac{1}{p}}$ using Lemma 2.2 and (2.3), and we bound $\frac{1}{1-2^{-\frac{1}{p}}}$ by $C p$, where $C$ is an absolute constant. We then multiply by $2^{-j}$ and take the supremum over $j \geq 0$, which gives

$$
\sup _{j \geq 0} 2^{-j}\left\|\left[\dot{\Delta}_{j}, T_{v_{n}} \partial_{m}\right] \bar{\omega}_{n}(t)\right\|_{L^{\infty}} \leq C p\left\|\bar{v}_{n}(t)\right\|_{\dot{B}_{\infty, \infty}^{0}}^{\frac{1}{q}} .
$$

For the case $j<0$, we follow identically the argument in [3], which yields the estimate

$$
\sup _{j<0} 2^{-j} \sum_{m=1}^{2}\left\|\left[\dot{\Delta}_{j}, T_{v_{n}} \partial_{m}\right] \bar{\omega}_{n}\right\|_{L^{\infty}} \leq C\|v\|_{L^{\infty}}\left\|\bar{v}_{n}\right\|_{L^{\infty}} .
$$

We now bound $\|v\|_{L^{\infty}}$ using Remark 2.1. We estimate the $L^{\infty}$-norm of $\bar{v}_{n}$ as in (4.3) and we apply (4.6) to conclude that

$$
\sup _{j<0} \sum_{m=1}^{2} 2^{-j}\left\|\left[\dot{\Delta}_{j}, T_{v_{n}} \partial_{m}\right] \bar{\omega}_{n}(t)\right\|_{L^{\infty}} \leq C 2^{-n}+C p\left\|\bar{v}_{n}(t)\right\|_{\dot{B}_{\infty, \infty}^{0}}^{\frac{1}{q}} .
$$

We now estimate the $L^{\infty}$-norm of $\left[\dot{\Delta}_{j}, T_{\partial_{m}} \cdot v_{n}\right] \bar{\omega}_{n}$. We write out the commutator and estimate the $L^{\infty}$-norm of $\dot{\Delta}_{j}\left(T_{\partial_{m} \bar{\omega}_{n}} v_{n}\right)$ and $T_{\partial_{m} \dot{\Delta}_{j} \bar{\omega}_{n}} v_{n}$ separately. By the definition of the paraproduct and by properties of our partition of unity, we have

$$
\begin{aligned}
& \left\|T_{\partial_{m} \dot{\Delta}_{j} \bar{\omega}_{n}} v_{n}\right\|_{L^{\infty}}=\left\|\sum_{l \geq 1} S_{l-1} \partial_{m} \dot{\Delta}_{j} \bar{\omega}_{n} \Delta_{l} v_{n}\right\|_{L^{\infty}} \\
\leq & \sum_{l=\max \{1, j\}}^{\infty}\left\|S_{l-1} \partial_{m} \dot{\Delta}_{j} \bar{\omega}_{n} \Delta_{l} v_{n}\right\|_{L^{\infty}} \leq C\left\|\dot{\Delta}_{j} \bar{\omega}_{n}\right\|_{L^{\infty} \sup _{l \geq 1}\left\|\Delta_{l} \nabla v_{n}\right\|_{L^{\infty}}} \\
\leq & C 2^{j}\left\|\dot{\Delta}_{j} \bar{v}_{n}\right\|_{L^{\infty} \sup _{l \geq 1}\left\|\Delta_{l} \nabla v_{n}\right\|_{L^{\infty}}}
\end{aligned}
$$

where we applied Bernstein's Lemma and took the sum to get the second inequality. We multiply (4.12) by $2^{-j}$, and we take the supremum over $j \in \mathbb{Z}$. This yields

$$
\sup _{j \in \mathbb{Z}} 2^{-j}\left\|T_{\partial_{m} \dot{\Delta}_{j} \bar{\omega}_{n}} v_{n}(t)\right\|_{L^{\infty}} \leq C\left\|\bar{v}_{n}(t)\right\|_{L^{\infty}}\left\|v_{n}(t)\right\|_{C_{*}^{1}} \leq C\left\|\bar{v}_{n}(t)\right\|_{L^{\infty}},
$$

where we used Remark 2.1 to get the last inequality. We bound $\left\|\bar{v}_{n}\right\|_{L^{\infty}}$ using (4.3) and (4.6). We conclude that

$$
\sup _{j \in \mathbb{Z}} 2^{-j}\left\|T_{\partial_{m} \dot{\Delta}_{j} \bar{\omega}_{n}} v_{n}(t)\right\|_{L^{\infty}} \leq C 2^{-n}+C p\left\|\bar{v}_{n}(t)\right\|_{\dot{B}_{\infty, \infty}^{0}}^{\frac{1}{q}} .
$$

Moreover, by an argument identical to that in [3], we have for $j \geq 0$

$$
\left\|\dot{\Delta}_{j}\left(T_{\partial_{m} \bar{\omega}_{n}} v_{n}\right)\right\|_{L^{\infty}} \leq C 2^{j}\left\|\bar{v}_{n}\right\|_{L^{\infty}}\left\|\omega^{0}\right\|_{L^{\infty}} .
$$

For the case $j<0,\left\|\dot{\Delta}_{j}\left(T_{\partial_{m} \bar{\omega}_{n}} v_{n}\right)\right\|_{L^{\infty}}$ is identically 0 . Therefore (4.13) still holds. We again bound $\left\|\bar{v}_{n}\right\|_{L^{\infty}}$ using (4.3) and (4.6), we multiply (4.13) by $2^{-j}$, and we take the supremum over $j \in \mathbb{Z}$, which yields

$$
\sup _{j \in \mathbb{Z}} 2^{-j}\left\|\dot{\Delta}_{j}\left(T_{\partial_{m} \bar{\omega}_{n}} v_{n}\right)(t)\right\|_{L^{\infty}} \leq C 2^{-n}+C p\left\|\bar{v}_{n}(t)\right\|_{\dot{B}_{\infty, \infty}^{0}}^{\frac{1}{q}} .
$$


To estimate the remainder, we again expand the commutator and consider each piece separately. We break $v_{n}$ into a low-frequency term and high-frequency term, and we consider $\left\|\dot{\Delta}_{j}\left(\partial_{m} R\left(\left(I d-S_{2}\right) v_{n}, \bar{\omega}_{n}\right)\right)\right\|_{L^{\infty}}$. We have

$$
\begin{array}{rl} 
& \sup _{j \in \mathbb{Z}} 2^{-j}\left\|\dot{\Delta}_{j}\left(\partial_{m} R\left(\left(I d-S_{2}\right) v_{n}, \bar{\omega}_{n}\right)\right)\right\|_{L^{\infty}} \\
\leq & C \sum_{l \geq 0} \sum_{i=-1}^{1}\left\|\Delta_{l-i}\left(I d-S_{2}\right) v_{n}\right\|_{L^{\infty}}\left\|\Delta_{l} \bar{\omega}_{n}\right\|_{L^{\infty}} \\
\leq & C \sum_{l \geq 0} \sum_{i=-1}^{1} 2^{-(l-i)}\left\|\Delta_{l-i}\left(I d-S_{2}\right) \nabla v_{n}\right\|_{L^{\infty}} 2^{l}\left\|\Delta_{l} \bar{v}_{n}\right\|_{L^{\infty}}^{\frac{1}{q}}\left\|\Delta_{l} \bar{v}_{n}\right\|_{L^{\infty}}^{\frac{1}{p}} \\
\leq C & C \bar{v}_{n}\left\|_{\dot{B}_{\infty}^{0}, \infty}^{\frac{1}{q}} \sum_{l \geq 0} \sum_{i=-1}^{1} 2^{-(l-i)}\right\| \Delta_{l-i}\left(I d-S_{2}\right) \nabla v_{n}\left\|_{L^{\infty}} 2^{l} 2^{-\frac{l}{p}}\right\| \Delta_{l} \bar{\omega}_{n} \|_{L^{\infty}}^{\frac{1}{p}},
\end{array}
$$

where we repeatedly used Bernstein's Lemma. We use Lemma 3 of [3] and (2.3) to bound $\left\|\Delta_{l-i}\left(I d-S_{2}\right) \nabla v_{n}\right\|_{L^{\infty}}$ and $\left\|\Delta_{l} \bar{\omega}_{n}\right\|_{L^{\infty}}$ by $\left\|\omega^{0}\right\|_{L^{\infty}}$ and sum over $l$ to conclude that

$$
\sup _{j \in \mathbb{Z}} 2^{-j}\left\|\dot{\Delta}_{j}\left(\partial_{m} R\left(\left(I d-S_{2}\right) v_{n}, \bar{\omega}_{n}\right)\right)\right\|_{L^{\infty}} \leq C\left\|\bar{v}_{n}\right\|_{\dot{B}_{\infty, \infty}^{0}}^{\frac{1}{q}} \frac{1}{1-2^{-\frac{1}{p}}} \leq C p\left\|\bar{v}_{n}\right\|_{\dot{B}_{\infty, \infty}^{0}}^{\frac{1}{q}} .
$$

To bound the low frequencies, we again apply an estimate established in [3], given by

$$
\sup _{j \in \mathbb{Z}} 2^{-j}\left\|\dot{\Delta}_{j}\left(\partial_{m} R\left(S_{2} v_{n}, \bar{\omega}_{n}\right)\right)\right\|_{L^{\infty}} \leq C\|v\|_{L^{\infty}}\left\|\bar{v}_{n}\right\|_{L^{\infty}} .
$$

We now bound $\|v\|_{L^{\infty}}$ using Remark 2.1 and we bound $\left\|\bar{v}_{n}\right\|_{L^{\infty}}$ using (4.3) and (4.6). This yields

$$
\sup _{j \in \mathbb{Z}} 2^{-j}\left\|\dot{\Delta}_{j}\left(\partial_{m} R\left(S_{2} v_{n}, \bar{\omega}_{n}\right)\right)(t)\right\|_{L^{\infty}} \leq C 2^{-n}+C p\left\|\bar{v}_{n}\right\|_{\dot{B}_{\infty, \infty}^{0}}^{\frac{1}{q}} .
$$

It remains to bound $\left.\sup _{j \in \mathbb{Z}} 2^{-j} \| \partial_{m} R\left(v_{n}, \dot{\Delta}_{j} \bar{\omega}_{n}\right)\right) \|_{L^{\infty}}$. Again we break $v_{n}$ into a lowfrequency and high-frequency term. We first estimate the high-frequency term. We reintroduce the sum over $m$ and utilize the divergence-free property of $v$ to put the partial derivative $\partial_{m}$ on $\bar{\omega}_{n}$. We then apply Bernstein's Lemma to conclude that for any fixed $j \in \mathbb{Z}$,

$$
\begin{aligned}
& \left.\sum_{m} \| R\left(\left(I d-S_{0}\right) v_{n}^{m}, \dot{\Delta}_{j} \partial_{m} \bar{\omega}_{n}\right)\right)\left\|_{L^{\infty}} \leq C \sum_{|k-l| \leq 1} 2^{l-k}\right\| \Delta_{k} \nabla v_{n}\left\|_{L^{\infty}}\right\| \dot{\Delta}_{j} \Delta_{l} \bar{\omega}_{n} \|_{L^{\infty}} \\
\leq & C \sup _{k \geq-1}\left\|\Delta_{k} \nabla v_{n}\right\|_{L^{\infty}}\left\|\dot{\Delta}_{j} \bar{\omega}_{n}\right\|_{L^{\infty}} \leq\|v\|_{C_{*}^{1}} 2^{j}\left\|\bar{v}_{n}\right\|_{L^{\infty}}
\end{aligned}
$$

The second inequality above follows because for fixed $j \geq 0$, we are summing only over $l$ satisfying $|l-j| \leq 1$, while for fixed $j<0$, we are only considering $l$ satisfying $-1 \leq l \leq 1$. We now multiply (4.14) by $2^{-j}$, and we take the supremum over $j \in \mathbb{Z}$, which yields

$$
\begin{aligned}
& \left.\sup _{j \in \mathbb{Z}} 2^{-j} \sum_{m} \| \partial_{m} R\left(\left(I d-S_{0}\right) v_{n}^{m}, \dot{\Delta}_{j} \bar{\omega}_{n}\right)\right)(t)\left\|_{L^{\infty}} \leq C\right\| \bar{v}_{n}(t) \|_{L^{\infty}} \\
\leq & C 2^{-n}+C p\left\|\bar{v}_{n}(t)\right\|_{\dot{B}_{\infty, \infty}^{0}}^{\frac{1}{q}},
\end{aligned}
$$


where we used (4.3) and (4.6) to bound $\left\|\bar{v}_{n}(t)\right\|_{L^{\infty}}$, and we used Remark 2.1 to bound $\|v(t)\|_{C_{*}^{1}}$. For the low-frequency term, we use the following estimate proved in [3]:

$$
\sup _{j \in \mathbb{Z}} 2^{-j} \sum_{m}\left\|\partial_{m} R\left(S_{0} v_{n}^{m}, \dot{\Delta}_{j} \bar{\omega}_{n}\right)\right\|_{L^{\infty}} \leq C\|v\|_{L^{\infty}}\left\|\bar{v}_{n}\right\|_{L^{\infty}} .
$$

As with previous terms, we use Remark 2.1 to bound $\|v\|_{L^{\infty}}$ and we use (4.3) combined with (4.6) to bound $\left\|\bar{v}_{n}\right\|_{L^{\infty}}$. We conclude that

$$
\sup _{j \in \mathbb{Z}} 2^{-j} \sum_{m}\left\|\partial_{m} R\left(S_{0} v_{n}^{m}, \dot{\Delta}_{j} \bar{\omega}_{n}\right)(t)\right\|_{L^{\infty}} \leq C 2^{-n}+C p\left\|\bar{v}_{n}(t)\right\|_{\dot{B}_{\infty, \infty}^{0}}^{\frac{1}{q}} .
$$

This completes the proof of Lemma 4.1.

Combining (4.7) with (4.8) and using the property that $A \geq 1$ gives

$$
\begin{aligned}
\partial_{t} \delta_{n}(t) & \leq C\left(2^{-n \alpha}+p \delta_{n}(t)^{\frac{1}{q}}+\frac{1}{A} p\left\|\bar{v}_{n}(t)\right\|_{\dot{B}_{\infty, \infty}^{0}}^{\frac{1}{q}}\right) \\
& \leq C\left(2^{-n \alpha}+p \delta_{n}(t)^{\frac{1}{q}}\right) .
\end{aligned}
$$

To complete the proof we closely follow an $\operatorname{argument}$ in [1]. We let $p=2-\log \delta_{n}(t)$ so that (4.15) reduces to

$$
\partial_{t} \delta_{n}(t) \leq C\left(2^{-n \alpha}+\left(2-\log \delta_{n}(t)\right)\left(\delta_{n}(t)\right)^{1-\frac{1}{2-\log \delta_{n}(t)}}\right) .
$$

We now observe that

$$
\delta_{n}(t)^{1-\frac{1}{2-\log \delta_{n}(t)}} \leq \delta_{n}(t)^{1+\frac{1}{\log \delta_{n}(t)}} \leq C \delta_{n}(t),
$$

where we used the equality $\delta_{n}(t)^{\frac{1}{\log \delta_{n}(t)}}=e$ to obtain the last inequality. We then see that (4.16) reduces to

$$
\partial_{t} \delta_{n}(t) \leq C\left(2^{-n \alpha}+\left(2-\log \delta_{n}(t)\right) \delta_{n}(t)\right) .
$$

Defining the function $\mu$ by $\mu(r)=r(2-\log r)$ and integrating with respect to time, we obtain

$$
\begin{aligned}
\delta_{n}(t) & \leq \delta_{n}(0)+C 2^{-n \alpha} t+C \int_{0}^{t} \mu\left(\delta_{n}(s)\right) d s \\
& \leq\left\|\bar{v}_{n}(0)\right\|_{\dot{B}_{\infty, \infty}^{0}}+C 2^{-n \alpha} t+C \int_{0}^{t} \mu\left(\delta_{n}(s)\right) d s,
\end{aligned}
$$

where we used that $\delta_{n}(0)=\frac{\left\|\bar{v}_{n}(0)\right\|_{\dot{B}_{\infty, \infty}^{0}}}{A} \leq\left\|\bar{v}_{n}(0)\right\|_{\dot{B}_{\infty, \infty}^{0}}$ since $A \geq 1$. We use the embedding $L^{\infty} \hookrightarrow \dot{B}_{\infty, \infty}^{0}$, the definition of $\bar{v}_{n}$, and Bernstein's Lemma to conclude that

$$
\left\|\bar{v}_{n}(0)\right\|_{\dot{B}_{\infty, \infty}^{0}} \leq C|| \bar{v}_{n}(0)\left\|_{L^{\infty}} \leq C \sum_{j \geq n} 2^{-j}\right\| \Delta_{j} \omega^{0}\left\|_{L^{\infty}} \leq C 2^{-n}\right\| \omega^{0} \|_{L^{\infty}} .
$$

We combine (4.18) with (4.17). This yields

$$
\delta_{n}(t) \leq C 2^{-n \alpha}(t+1)+C \int_{0}^{t} \mu\left(\delta_{n}(s)\right) d s .
$$


We now recall Osgood's Lemma. A proof of the lemma can be found in [2].

Lemma 4.2. Let $\rho$ be a positive borelian function, and let $\gamma$ be a locally integrable positive function. Assume that, for some strictly positive number $\beta$, the function $\rho$ satisfies

$$
\rho(t) \leq \beta+\int_{t_{0}}^{t} \gamma(s) \mu(\rho(s)) d s
$$

Then

$$
-\phi(\rho(t))+\phi(\beta) \leq \int_{t_{0}}^{t} \gamma(s) d s
$$

where $\phi(x)=\int_{x}^{1} \frac{1}{\mu(r)} d r$.

We recall that we are working on a fixed time interval $[0, T]$ and we assume $n$ is large enough to ensure that $C 2^{-n \alpha}(T+1) \leq e^{2}$. We let $\phi, \rho, \gamma$, and $\beta$ be given by the following:

$\phi(x)=\log (2-\log x)-\log 2, \rho(t)=\delta_{n}(t), \gamma(t)=C$, and $\beta=C 2^{-n \alpha}(T+1)$.

Applying Osgood's Lemma, we have that for any $t \leq T$,

$$
-\log \left(2-\log \delta_{n}(t)\right)+\log \left(2-\log \left(C 2^{-n \alpha}(T+1)\right) \leq C t .\right.
$$

Taking the exponential twice gives

$$
\delta_{n}(t) \leq e^{2-2 e^{-C t}}\left(C(T+1) 2^{-n \alpha}\right)^{e^{-C t}} .
$$

Multiplying both sides by $A$ yields the result. This completes the proof of Lemma 3.3 .

\section{REFERENCES}

[1] J.Y. Chemin, A remark on the inviscid limit for two-dimensional incompressible fluids, Commun. Part. Differ. Equs., 21(11,12), 1771-1779, 1996.

[2] J.Y. Chemin, Perfect Incompressible Fluids, Oxford Lecture Series in Mathematics and its Applications, Clarendon Press, Oxford, 14, 1998.

[3] E. Cozzi, Vanishing viscosity in the plane for nondecaying velocity and vorticity, SIAM J. Math. Anal., 41(2), 495-510, 2009.

[4] J. Duchon and R. Robert, Inertial energy dissipation for weak solutions of incompressible Euler and Navier-Stokes equations, Nonlinearity, 13, 249-255, 2000.

[5] T. Hmidi, Régularité Höldérienne des poches de tourbillon visqueuses, J. Math. Pures Appl., 84, 1455-1495, 2005.

[6] O. Sawada and Y. Taniuchi, A remark on $L^{\infty}$ solutions to the 2-D Navier-Stokes equations, J. Math. Fluid Mech., 9(4), 533-542, 2007.

[7] P. Serfati, Solutions $C^{\infty}$ en temps, $n$-log Lipschitz bornées en espace et équation d'Euler, C.R. Acad. Sci. Paris, 320(I), 555-558, 1995.

[8] V. Yudovich, Nonstationary flows of an ideal incompressible fluid, Zhurnal Vych Matematika, 3, 1032-1066, 1963. 